\title{
IUFOST2006/705 \\ Rapid Methods and Automation in Microbiology:25 years of Scien- tific Developments and Global Market Trends
}

\author{
D. Fung \\ Kansas State University, 207 Call Hall, Manhattan, 66506, United States of America \\ dfung@ksu.edu
}

\begin{abstract}
Rapid Methods and Automation in Microbiology is a dynamic area in microbiology dealing with the study of improved methods in the early detection, isolation, characterization and enumeration of microorganisms and their products in clinical, drugs, industrial, food, and environmental samples. Rapidly monitoring the presence, growth characteristics, and product developments of benefical and pathogenic microorganisms in the food supply are important in global movement of food products and commodaties. This presentation will focus on the developments of the field in the past 25 years on sample preparations using ingenious methods, rapid viable cell counts involving new technologies, miniaturized microbiology and effective diagnostic tests and kits, rapid, effective, and automated immunological tests, advanced instrumentations concerning physical, chemical, anad biochemical methods, genetic methods, Polymerase Chain Reactions, Ribotyping, biosensers, microarrays, etc. Global trends in the volume and kinds of tests being conducted for foods (meats, vegetables, dairy, processed foods), water, pharmcueticals, personal care products, etc. will be made. Market value of these tests internationally from 1998 to 2010 will be discussed. The presentation will conclude with a discussion on Fung's 10 predictions into the future of the total picture of microbiological testings for food and consumer safety.
\end{abstract}

